\title{
A Comparison Principle for the Mean Curvature Flow Equation with Discontinuous Coefficients
}

\author{
Cecilia De Zan and Pierpaolo Soravia \\ Dipartimento di Matematica, Università di Padova, Via Trieste 63, 35121 Padova, Italy \\ Correspondence should be addressed to Pierpaolo Soravia; soravia@math.unipd.it
}

Received 29 April 2016; Accepted 14 July 2016

Academic Editor: Yoshikazu Giga

Copyright (C) 2016 C. De Zan and P. Soravia. This is an open access article distributed under the Creative Commons Attribution License, which permits unrestricted use, distribution, and reproduction in any medium, provided the original work is properly cited.

We study the level set equation in a bounded domain when the velocity of the interface is given by the mean curvature plus a discontinuous velocity. We prove a comparison principle for the initial-boundary value problem whose consequence is uniqueness of continuous solutions and well- posedness of the level set method.

\section{Introduction}

We consider the Dirichlet initial-boundary value problem for a mean curvature flow equation

$$
\begin{aligned}
& u_{t}(x, t)+f(x)|D u(x, t)|+F\left(D u(x, t), D^{2} u(x, t)\right) \\
& =0, \quad(x, t) \in \Omega \times(0,+\infty), \\
& u(x, 0)=u_{o}(x) \in C(\Omega), \\
& u(x, t)=g(x, t), \quad(x, t) \in \partial \Omega \times[0,+\infty),
\end{aligned}
$$

where $\Omega \subset \mathbb{R}^{n}$ is open and bounded, $F: \mathbb{R}^{n} \backslash\{0\} \times \delta^{n} \rightarrow \mathbb{R}$ is the standard mean curvature operator

$$
F(p, X)=-\operatorname{tr}\left[\left(I-\frac{p}{|p|} \otimes \frac{p}{|p|}\right) X\right]
$$

$\delta^{n}$ denotes the space of the $n \times n$ symmetric matrices, and the function $f: \Omega \rightarrow \mathbb{R}$ is piecewise Lipschitz continuous across Lipschitz hypersurfaces. Since (1) is nonlinear, it has a singularity at $D u=0$ and discontinuous coefficients; then the appropriate notion of solution is that of viscosity solution as in Crandall et al. [1]. We will address uniqueness of solutions of (1) by proving a comparison principle. The consequence will be uniqueness of continuous solutions of (1). At the present time we do not have existence results for continuous solutions although the coercivity of the norm and the regularizing effect of the mean curvature operator appearing in the equation lead to conjecture that this should be the case.

Equation (1) appears in the study of the weak front evolution of hypersurfaces with a given normal velocity. It appears, for instance, in the study of the refraction phenomenon in anisotropic media with a discontinuous refraction index or in the study of phase transitions occurring in singular perturbation problems for nonlinear parabolic equations. The main novelty of the paper is that we address the problem when the coefficient $f$ may be discontinuous while in the literature $f$ is always assumed to be continuous.

One can easily observe that if $u$ solves the pde in (1) and $\psi: \mathbb{R} \rightarrow \mathbb{R}$ is smooth and increasing, then, at least formally, also $\psi(u)$ solves the same pde. Hence the equation is called geometric. As a consequence, if $u_{o}^{1}$ and $u_{o}^{2}$ are two initial conditions such that

$$
\Lambda_{o}=\left\{x: u_{o}^{1}(x)=0\right\}=\left\{x: u_{o}^{2}(x)=0\right\}
$$

is a closed hypersurface and comparison principle holds, then if $u^{1}, u^{2}$ are the corresponding solutions of (1), one shows that

$$
\left\{x: u^{1}(x, t)=0\right\}=\Lambda_{t}=\left\{x: u^{2}(x, t)=0\right\},
$$

for $t>0$.

We can define the family $\left(\Lambda_{t}\right)_{t}$ as the geometric flow of the hypersurface $\Lambda_{o}$ with discontinuous normal velocity 
$(\kappa+f(x)) \mathbf{n}$ in the unisotropic medium, $\kappa$ being the mean curvature of the front and $\mathbf{n}$ its normal vector. This is the key observation of the level set method for weak front propagation introduced by Osher and Sethian [2].

The theory of viscosity solutions for Hamilton-Jacobi equations with discontinuous coefficients has now a rather long history. The problem was first studied by Ishii [3] who defined viscosity solutions for Hamiltonians discontinuous in the variables $(t, u)$. In the case that discontinuities of the coefficients concern the state variable $x$, the problem is quite interesting and more delicate. In order to have well-posed problems, one needs some strong structure of the equation either (i) to strengthen the classical notion of viscosity solution or (ii) to strongly limit the class of allowed discontinuities. Within the first group of papers we recall the work on uniformly elliptic/parabolic pdes by Jensen [4] and by Caffarelli et al. [5]; see also the references therein. For firstorder, convex, and coercive Hamiltonians (i.e., $H(x, p) \rightarrow$ $+\infty$ as $|p| \rightarrow \infty)$ uniqueness results have been obtained by Camilli and Siconolfi [6]. Other studies on more general classes of equations, in particular degenerate and noncoercive Hamiltonians, using the standard notion of viscosity solution, are limited to piecewise continuous coefficients. Our problem fits into this category. We recall previous work of one of the authors on the characterization of uniqueness of viscosity solutions [7], existence and uniqueness results for the stationary eikonal equation [8], and general uniqueness results for degenerate elliptic equations [9]; see however also the references therein for additional work on the subject. For more recent works, in the case of time dependent first order problems also including equations of evolving interfaces, we recall the paper of the authors [10] on the evolution equation for the eikonal equation as well as Chen and $\mathrm{Hu}$ [11], Deckelnick and Elliott [12], Barles et al. [13, 14], Bressan and Hong [15], and Coclite and Risebro [16].

\section{The Level Set Approach}

In this section we briefly recall the main ideas of the weak evolution of interfaces named the level set approach. Let $\Lambda_{t}$ be a generic interface at time $t>0$; we suppose that $\Lambda_{t}$ is the topological boundary of an open set $D_{t} \subset \mathbb{R}^{n}$, that is, $\Lambda_{t}=\partial D_{t} \subset \mathbb{R}^{n}$. Assume moreover that, for any point $x \in \mathbb{R}^{n}$, the exterior normal vector $\mathbf{n}(x)$ at $x \in D_{t}$ is well defined and smooth and that $x$ moves with normal velocity

$$
V=v(x, t, \mathbf{n}(x), D \mathbf{n}(x)) .
$$

A classical problem is the following: given $D_{0} \subset \mathbb{R}^{n}$ open, study the evolution of the interfaces $t \mapsto \Lambda_{t}$ moving with normal velocity $V=v(x, t, D \mathbf{n}(x), \mathbf{n}(x))$ and starting at time $t=0$ from $\Lambda_{0}=\partial D_{0}$. One of the main difficulties in interface dynamics with normal velocity as in (5) is that they develop singularities in finite time, independently of the smoothness of the initial surface $\Lambda_{0}$. To interpret the evolution past the singularities it is appropriate to use the theory of viscosity solutions for partial differential equations. The so-called level set approach, based on viscosity solutions, was first developed for the mean curvature flow equation independently by Evans and Spruck in [17] and by Chen et al. in [18] for more general geometric equations. These works were later extended by Ishii and Souganidis in [19] and by Goto in [20] for more general motions and more general initial surfaces. For a detailed analysis of this approach we refer the reader to the book by Giga [21], Souganidis [22], and the references therein. We now briefly discuss the main ideas.

Problem (5) can be reformulated in an equivalent way. Suppose that there exists a smooth function $u: \mathbb{R}^{n} \times$ $[0,+\infty) \rightarrow \mathbb{R}$ such that

$$
\begin{aligned}
& \Lambda_{t}=\left\{x \in \mathbb{R}^{n}: u(x, t)=0\right\}, \\
& D_{t}=\left\{x \in \mathbb{R}^{n}: u(x, t)>0\right\},
\end{aligned}
$$

$D u \neq 0$ on $\Lambda_{t}$

it can be easily seen that

$$
\begin{aligned}
V & =\frac{u_{t}}{|D u|}, \\
\mathbf{n} & =-\frac{D u}{|D u|} \\
D \mathbf{n} & =-\frac{1}{|D u|}\left(I-\frac{D u \otimes D u}{|D u|^{2}}\right) D^{2} u
\end{aligned}
$$

and so (5) becomes

$$
u_{t}=F\left(x, t, D u, D^{2} u\right)
$$

with $F$ defined as

$$
\begin{aligned}
& F(x, t, p, X) \\
& =|p| v\left(x, t,-\frac{p}{|p|},-\frac{1}{|p|}\left(I-\frac{p \otimes p}{|p|^{2}}\right) X\right), \\
& (x, t, p, X) \in \mathbb{R}^{n} \times(0,+\infty) \times \mathbb{R}^{n} \times \mathcal{S}^{n} .
\end{aligned}
$$

This means that $F$ is as smooth as $v$ with possible singularity at $p=0$ and that $F$ is geometric; that is, it satisfies, for any $(x, t) \in \mathbb{R}^{n} \times(0,+\infty), p \in \mathbb{R}^{n}$ and $X \in \mathcal{S}^{n}$,

$$
\begin{aligned}
& F(x, t, \lambda p, \lambda X+\mu(p \otimes p))=\lambda F(x, t, p, X) \\
& \forall \lambda>0, \mu \in \mathbb{R} .
\end{aligned}
$$

The so-called level set approach wants to solve the interface evolution equation (5) starting at a given $\Lambda_{0}=\partial D_{0}$ looking at the (viscosity) solutions of (8). To do this we take an auxiliary function $u_{0}: \mathbb{R}^{n} \rightarrow \mathbb{R}$, at least continuous and such that

$$
\begin{aligned}
& \Lambda_{0}=\left\{x \in \mathbb{R}^{n}: u_{0}(x)=0\right\}, \\
& D_{0}=\left\{x \in \mathbb{R}^{n}: u_{0}(x)>0\right\} .
\end{aligned}
$$

Once function $u_{0}$ is chosen one solves (8) with initial data $u(x, 0)=u_{0}(x)$ and defines for any $t>0$ and such a solution $u$

$$
\begin{aligned}
& \Lambda_{t}=\left\{x \in \mathbb{R}^{n}: u(x, t)=0\right\}, \\
& D_{t}=\left\{x \in \mathbb{R}^{n}: u(x, t)>0\right\} .
\end{aligned}
$$


In order to consider the collection of pairs $\left(\Lambda_{t}, D_{t}\right)_{t \geq 0}$ as a generalized solution of our evolution problem with initial data $\left(\Lambda_{0}, D_{0}\right)$ it is crucial to prove that, under suitable hypothesis, $\left(\Lambda_{t}, D_{t}\right)_{t \geq 0}$ depends only on $\left(\Lambda_{0}, D_{0}\right)$ and not on the particular chosen function $u_{0}$. Key properties on $F$ to this end are ellipticity of $F$, that is,

$$
F(x, t, p, X) \leq F(x, t, p, Y), \quad \text { if } X \geq Y,
$$

the fact that $F$ is geometric as described above, the technical fact that $F^{*}(x, t, 0,0)=F_{*}(x, t, 0,0)$, for all $(x, t) \in \mathbb{R}^{n} \times$ $(0,+\infty)$, and a comparison principle for pde (8). Recall here that if $g: \Omega \rightarrow \mathbb{R}$ is a generic locally bounded function we define the upper semicontinuous envelope of $g$ as $g^{*}: \bar{\Omega} \rightarrow \mathbb{R}$,

$$
g^{*}(x)=\lim _{r \rightarrow 0^{+}} \sup _{|y-x|<r, y \in \Omega} g(y)=\inf _{r>0} \sup _{|y-x|<r, y \in \Omega} g(y),
$$

and the lower semicontinuous envelope of $g$ as

$$
g_{*}(x)=\lim _{r \rightarrow 0^{+}} \inf _{|y-x|<r, y \in \Omega} g(y)=\sup _{r>0} \inf _{|y-x|<r, y \in \Omega} g(y) .
$$

Indeed one can prove that (8) is invariant by nondecreasing changes of variable $u \mapsto \theta(u)$ and the weak evolution is well defined. Case (1) that we consider in the present paper is a notable example of the theory in that it contains the mean curvature operator and adds a discontinuous coefficient in the space variable which is a novelty in the theory.

We end this section by recalling the definition of viscosity solution for our equation, due to Ishii.

Definition 1. Let $\Omega \subset \mathbb{R}^{n}$ be an open set.

(i) An upper semicontinuous function $u: \Omega \times(0,+\infty) \rightarrow$ $\mathbb{R}$ is a viscosity subsolution of the differential equation in (1) if for every $\varphi \in C^{2}(\Omega \times(0,+\infty))$ and for every local maximum point $(x, t) \in \Omega \times(0,+\infty)$ of $u-\varphi$, we have

$$
\begin{aligned}
\varphi_{t}(x, t) & +f_{*}(x)|D \varphi(x, t)| \\
& +F_{*}\left(D \varphi(x, t), D^{2} \varphi(x, t)\right) \leq 0 .
\end{aligned}
$$

One calls $\varphi$ a test function at $(x, t)$ for the subsolution $u$. Similarly, one has the following.

(ii) A lower semicontinuous function $u: \Omega \times(0,+\infty) \rightarrow$ $\mathbb{R}$ is a viscosity supersolution of the differential equation in (1) if and only if for every $\varphi \in C^{2}(\Omega \times(0,+\infty))$ and for every local minimum point $(x, t) \in \Omega \times(0,+\infty)$ of $u-\varphi$, we have

$$
\begin{aligned}
\varphi_{t}(x, t) & +f^{*}(x)|D \varphi(x, t)| \\
& +F^{*}\left(D \varphi(x, t), D^{2} \varphi(x, t)\right) \geq 0 .
\end{aligned}
$$

(iii) A locally bounded function $u: \Omega \times(0,+\infty) \rightarrow \mathbb{R}$ is a viscosity solution of the differential equation in (1) if and only if $u^{*}$ is a viscosity subsolution and $u_{*}$ is a viscosity subsolution.

\section{The Comparison Principle}

As we mentioned in the Introduction, we will consider only special discontinuities in the coefficient $f$ and we now make this precise. The function $f: \Omega \rightarrow \mathbb{R}$ will be piecewise Lipschitz continuous across a Lipschitz hypersurface according to the following definition.

Definition 2. One says that $f: \Omega \rightarrow \mathbb{R}$ is a piecewise Lipschitz continuous function across a Lipschitz hypersurface if the discontinuity set $\Gamma \subset \Omega$ of $f$ satisfies the following properties. One can partition $\Omega$ like

$$
\Omega=\Omega^{+} \cup \Omega^{-} \cup \Gamma
$$

where $\Omega^{ \pm}$are nonempty, open, and connected (the two sides of $\Gamma$ ). Moreover, $f$ is locally Lipschitz continuous in $\Omega \backslash \Gamma$; $f$ has a Lipschitz continuous extension in $\Omega^{+} \cup \Gamma$ (i.e., $f^{+}$) and in $\Omega^{-} \cup \Gamma$ (i.e., $f^{-}$):

$$
f^{*}(x)=f^{+}(x)>f^{-}(x)=f_{*}(x), \quad \forall x \in \Gamma .
$$

For all $x \in \Gamma$ we have $f(x) \in\left[f_{*}(x), f^{*}(x)\right]$.

Technically crucial although straightforward in what follows is the next remark.

Remark 3. Suppose that $f$ is piecewise continuous as in Definition 2 and let $\Gamma$ be the Lipschitz continuous hypersurface of its discontinuities. Then it is not hard to show that we can always find unit vectors $\eta^{+}, \eta^{-} \in \mathbb{R}^{n}$ inward $\Omega^{+}, \Omega^{-}$, respectively, at every $\widehat{x} \in \Gamma$. This means that for some $h, c>0$ we have that $B\left(y+t \eta^{+}, t c\right) \subset \Omega^{+}$for all $y \in B(\widehat{x}, h) \cap \bar{\Omega}^{+}$, $t \in(0, c)$ and for all $\hat{x} \in \Gamma$ and that $B\left(y+t \eta^{-}, t c\right) \subset \Omega^{-}$for all $y \in B(\widehat{x}, h) \cap \bar{\Omega}, t \in(0, c)$ and for all $\hat{x} \in \Gamma$. We may also suppose that $\eta^{-}=-\eta^{+}$. For this straightforward computation see, for instance, [23].

If moreover we suppose that $\Gamma$ is globally the graph of a Lipschitz continuous function of $n-1$ variables with $L_{f}>$ 0 as a Lipschitz constant then the vectors $\eta^{ \pm}$may be chosen independent of the point $\hat{x}$.

We will indicate below convenience $\Omega_{\infty}=\Omega \times(0,+\infty)$, and the parabolic boundary $\partial \Omega_{\infty}=(\Omega \times\{0\}) \cup(\partial \Omega \times[0,+\infty))$. We are studying the equation

$$
\begin{aligned}
& u_{t}(x, t)+f(x)|D u(x, t)|+F\left(D u(x, t), D^{2} u(x, t)\right) \\
& \quad=0, \quad(x, t) \in \Omega_{\infty},
\end{aligned}
$$

where $F: \mathbb{R}^{n} \backslash\{0\} \times \mathcal{S}^{n} \rightarrow \mathbb{R}$ is the mean curvature operator (see (2)).

We now state and prove our comparison result for (20) in $\Omega_{\infty}$, when $\Omega \subset \mathbb{R}^{n}$ is open and bounded. The techniques used in this proof are a combination of the classical ideas of Chen et al. [18] (see also the book by Giga [21]) to treat the singularity of the mean curvature term at $p=0$ and the techniques developed by one of the authors [9] to prove uniqueness of viscosity solutions of degenerate second-order Hamilton-Jacobi equations with discontinuous coefficients.

Theorem 4. Let $\Omega \subset \mathbb{R}^{n}$ be open and bounded. Assume that $f: \Omega \rightarrow \mathbb{R}$ is a piecewise continuous function across a Lipschitz graph $\Gamma$. Let $u, v: \bar{\Omega}_{\infty} \rightarrow \mathbb{R}$ be, respectively, an upper 
semicontinuous subsolution and a continuous supersolution of (20) in $\Omega_{\infty}$. If $u \leq v$ on $\partial \Omega_{\infty}$, then $u \leq v$ on $\Omega_{\infty}$.

Before going to the proof we just observe that in the statement the roles of $u, v$ can be interchanged; that is, $u$ can be a continuous subsolution and $v$ a lower semicontinuous supersolution of (20) in $\Omega_{\infty}$.

Proof. Since (20) is invariant by an increasing change of the dependent variable, it is not restrictive to assume that $u$ and $v$ are bounded by taking $\tanh u, \tanh v$ instead of $u, v$.

Assume by contradiction that there exists a point $\left(x_{o}, t_{o}\right) \in \Omega_{\infty}$ such that $u\left(x_{o}, t_{o}\right)-v\left(x_{o}, t_{o}\right)=A>0$. Fix any $\alpha \in \mathbb{R}, 0<\alpha<A / t_{o}$ and let $(\hat{x}, \widehat{t})$ be a maximum point of

$$
\Phi(x, t):=u(x, t)-v(x, t)-\alpha t, \quad(x, t) \in \bar{\Omega}_{\infty} .
$$

Clearly the maximum of $\Phi$ exists since $\Omega$ is bounded, $u-v$ is upper semicontinuous, $\Phi\left(x_{o}, t_{o}\right)=A-\alpha t_{o}>0, \Phi \leq 0$ on $\partial \Omega_{\infty}$, and $\Phi(x, t) \leq 0$ for large $t$ since $u, v$ are bounded. Notice that $\left(x_{o}, t_{o}\right) \notin \partial \Omega_{\infty}$ and $\max _{\bar{\Omega} \times[0,+\infty)} \Phi \geq \Phi\left(x_{o}, t_{o}\right)=$ $A-\alpha t_{o}=2 \gamma>0$; therefore every maximum point of $\Phi$ cannot be on $\partial \Omega_{\infty}$.

We now define

$$
\begin{aligned}
\omega^{\varepsilon}(x, y, t, s):= & u(x, t)-v(y, s)-\frac{\gamma}{4}\left|\frac{x-y}{\varepsilon}+\eta\right|^{4} \\
& -\frac{\gamma}{2}\left|\frac{t-s}{\varepsilon}\right|^{2}-\alpha t
\end{aligned}
$$

where $\eta=\eta^{-}=-\eta^{+}$is as in Remark 3, and consider $\left(x_{\varepsilon}, y_{\varepsilon}, t_{\varepsilon}, s_{\varepsilon}\right) \in \Omega_{\infty} \times \Omega_{\infty}$ such that

$$
\begin{aligned}
& \omega^{\varepsilon}\left(x_{\varepsilon}, y_{\varepsilon}, t_{\varepsilon}, s_{\varepsilon}\right) \\
& \quad=\max \left\{\omega^{\varepsilon}(x, y, t, s):(x, y, t, s) \in \bar{\Omega}_{\infty} \times \bar{\Omega}_{\infty}\right\} .
\end{aligned}
$$

As above the maximum of $\omega^{\varepsilon}$ exists and it is positive since

$$
\omega^{\varepsilon}(\widehat{x}, \widehat{x}, \widehat{t}, \widehat{t})=\Phi(\widehat{x}, \widehat{t})-\frac{\gamma}{4}|\eta|^{4} \geq 2 \gamma-\frac{\gamma}{4} \geq \gamma>0
$$

Moreover from $\omega^{\varepsilon}\left(x_{\varepsilon}, y_{\varepsilon}, t_{\varepsilon}, s_{\varepsilon}\right) \geq \omega^{\varepsilon}(\hat{x}, \hat{x}, \hat{t}, \hat{t})>0$ and the boundedness of $u, v$ we obtain that

$$
\begin{gathered}
\left|\frac{x_{\varepsilon}-y_{\varepsilon}}{\varepsilon}+\eta\right|, \\
\left|\frac{t_{\varepsilon}-s_{\varepsilon}}{\varepsilon}\right|
\end{gathered}
$$

are uniformly bounded in $\varepsilon>0$ since we have assumed that $u$ and $v$ are bounded, and we get that $\left|x_{\varepsilon}-y_{\varepsilon}\right|,\left|t_{\varepsilon}-s_{\varepsilon}\right| \rightarrow 0$ if $\varepsilon \rightarrow 0$. Therefore $\left(x_{\varepsilon}, t_{\varepsilon}\right),\left(y_{\varepsilon}, s_{\varepsilon}\right)$ stay in a bounded region uniformly in $\varepsilon>0$ and

$$
\lim _{\varepsilon \rightarrow 0^{+}}\left(x_{\varepsilon}, y_{\varepsilon}, t_{\varepsilon}, s_{\varepsilon}\right)=(\bar{x}, \bar{x}, \bar{t}, \bar{t}) \in \overline{\Omega_{\infty}} \times \overline{\Omega_{\infty}} .
$$

Using the upper semicontinuity of $u$ and the continuity of $v$ we compute

$$
\begin{aligned}
\Phi(\bar{x}, \bar{t}) & =u(\bar{x}, \bar{t})-v(\bar{x}, \bar{t})-\alpha \bar{t} \\
& \geq \limsup _{\varepsilon \rightarrow 0^{+}}\left(u\left(x_{\varepsilon}, t_{\varepsilon}\right)-v\left(y_{\varepsilon}, s_{\varepsilon}\right)-\alpha t_{\varepsilon}\right) \\
& \geq \limsup _{\varepsilon \rightarrow 0^{+}} \omega^{\varepsilon}\left(x_{\varepsilon}, y_{\varepsilon}, t_{\varepsilon}, s_{\varepsilon}\right) \\
& \geq \lim _{\varepsilon \rightarrow 0^{+}} \omega^{\varepsilon}(\widehat{x}, \widehat{x}+\varepsilon \eta, \widehat{t}, \widehat{t}) \\
& =\lim _{\varepsilon \rightarrow 0^{+}}(u(\widehat{x}, \widehat{t})-v(\widehat{x}+\varepsilon \eta, \widehat{t})-\alpha \widehat{t}) \\
& =\Phi(\widehat{x}, \widehat{t}) \geq \Phi(\bar{x}, \bar{t}) .
\end{aligned}
$$

From here we obtain that $(\bar{x}, \bar{t})$ is a maximum point for $\Phi$, so $(\bar{x}, \bar{t}) \in \Omega_{\infty}$ and moreover

$$
\lim _{\varepsilon \rightarrow 0^{+}} u\left(x_{\varepsilon}, t_{\varepsilon}\right)=u(\bar{x}, \bar{t}) .
$$

Expanding again

$$
\omega^{\varepsilon}(\bar{x}, \bar{x}+\varepsilon \eta, \bar{t}, \bar{t}) \leq \omega^{\varepsilon}\left(x_{\varepsilon}, y_{\varepsilon}, t_{\varepsilon}, s_{\varepsilon}\right) .
$$

By the continuity of $v$ and (28) we improve previous estimates as

$$
\begin{aligned}
\lim _{\varepsilon \rightarrow 0^{+}}\left|\frac{x_{\varepsilon}-y_{\varepsilon}}{\varepsilon}+\eta\right| & =0, \\
\lim _{\varepsilon \rightarrow 0^{+}}\left|\frac{t_{\varepsilon}-s_{\varepsilon}}{\varepsilon}\right| & =0 .
\end{aligned}
$$

Hence for $\varepsilon$ sufficiently small

$$
\left|x_{\varepsilon}-y_{\varepsilon}+\varepsilon \eta\right| \leq c \varepsilon,
$$

where $c$ appears in Remark 3. In particular (31) shows that if $\bar{x} \in \Gamma$ and $x_{\varepsilon} \in \Omega^{-} \cup \Gamma$, then $y_{\varepsilon} \in \Omega^{-}$by Remark 3 which is something that we keep in mind for later. Up to now we never used the equation. Now consider two different cases.

Case 1. Suppose that there exists a sequence $\varepsilon_{k} \rightarrow 0^{+}$such that $y_{\varepsilon_{k}}=x_{\varepsilon_{k}}+\varepsilon_{k} \eta$ for all $k$. We omit from now on the subindex $k$. Since $\omega^{\varepsilon}\left(x, y_{\varepsilon}, t, s_{\varepsilon}\right) \leq \omega^{\varepsilon}\left(x_{\varepsilon}, y_{\varepsilon}, t_{\varepsilon}, s_{\varepsilon}\right)$ for any $(x, t) \in \Omega_{\infty}$ we get

$$
\max _{\Omega_{\infty}}\left(u-\varphi^{+}\right)=u\left(x_{\varepsilon}, t_{\varepsilon}\right)-\varphi^{+}\left(x_{\varepsilon}, t_{\varepsilon}\right)
$$

where

$$
\varphi^{+}(x, t)=\frac{\gamma}{4}\left|\frac{x-y_{\varepsilon}}{\varepsilon}+\eta\right|^{4}+\frac{\gamma}{2}\left|\frac{t-s_{\varepsilon}}{\varepsilon}\right|^{2}+\alpha t .
$$

Similarly we see that

$$
\min _{\Omega_{\infty}}\left(v-\varphi^{-}\right)=v\left(y_{\varepsilon}, s_{\varepsilon}\right)-\varphi^{-}\left(y_{\varepsilon}, s_{\varepsilon}\right),
$$

with

$$
\varphi^{-}(y, s)=-\frac{\gamma}{4}\left|\frac{x_{\varepsilon}-y}{\varepsilon}+\eta\right|^{4}-\frac{\gamma}{2}\left|\frac{t_{\varepsilon}-s}{\varepsilon}\right|^{2} .
$$


Since we have assumed that $x_{\varepsilon}=y_{\varepsilon}+\varepsilon \eta$ we have $\left|D \varphi^{+}\left(x_{\varepsilon}, t_{\varepsilon}\right)\right|=\left|D^{2} \varphi^{+}\left(x_{\varepsilon}, t_{\varepsilon}\right)\right|=0$ and $\left|D \varphi^{-}\left(y_{\varepsilon}, s_{\varepsilon}\right)\right|=$ $\left|D^{2} \varphi^{-}\left(y_{\varepsilon}, s_{\varepsilon}\right)\right|=0$. And so, using the fact that $u$ is a viscosity subsolution and $v$ a supersolution of (20) we obtain by computing derivatives

$$
\alpha+\frac{\gamma}{\varepsilon^{2}}\left(t_{\varepsilon}-s_{\varepsilon}\right) \leq 0 \leq \frac{\gamma}{\varepsilon^{2}}\left(t_{\varepsilon}-s_{\varepsilon}\right),
$$

since here $F^{*}\left(y_{\varepsilon}, s_{\varepsilon}, 0,0\right)=F_{*}\left(x_{\varepsilon}, t_{\varepsilon}, 0,0\right)=0$. This contradicts $\alpha>0$.

Case 2. We now suppose that $x_{\varepsilon} \neq y_{\varepsilon}+\varepsilon \eta$ for $\varepsilon$ small enough. We set $\xi=(x, t), \zeta=(s, y)$, and

$$
\varphi(x, t, y, s)=\frac{\gamma}{4}\left|\frac{x-y}{\varepsilon}+\eta\right|^{4}+\frac{\gamma}{2}\left|\frac{t-s}{\varepsilon}\right|^{2} .
$$

We now use classical matter. Observe that since $(x, t, y, s) \mapsto$ $u(x, t)-v(y, s)-\alpha t-\varphi(x, t, y, s)$ takes its maximum over $\overline{\Omega_{\infty}} \times \overline{\Omega_{\infty}}$ at $\left(x_{\varepsilon}, t_{\varepsilon}, y_{\varepsilon}, s_{\varepsilon}\right)$, we see that

$$
\left(\left(\begin{array}{c}
D_{\xi} \varphi\left(\xi_{\varepsilon}, \zeta_{\varepsilon}\right) \\
D_{\zeta} \varphi\left(\xi_{\varepsilon}, \zeta_{\varepsilon}\right)
\end{array}\right), A\right) \in J^{2,+}\left(u\left(\xi_{\varepsilon}\right)-v\left(\zeta_{\varepsilon}\right)-\alpha t_{\varepsilon}\right),
$$

the superjet of the indicated function, where

$$
A=\left(\begin{array}{cc}
D_{\xi \xi}^{2} \varphi\left(\xi_{\varepsilon}, \zeta_{\varepsilon}\right) & D_{\xi \zeta}^{2} \varphi\left(\xi_{\varepsilon}, \zeta_{\varepsilon}\right) \\
D_{\zeta \xi}^{2} \varphi\left(\xi_{\varepsilon}, \zeta_{\varepsilon}\right) & D_{\zeta \zeta}^{2} \varphi\left(\xi_{\varepsilon}, \zeta_{\varepsilon}\right)
\end{array}\right)
$$

Now we apply the theorem on sums (see [1]) to find that for every $\lambda>0$ there exist two matrices $X, Y \in \mathcal{S}^{n}$ such that

$$
\begin{gathered}
\left(\alpha+\varphi_{t}\left(\xi_{\varepsilon}, \zeta_{\varepsilon}\right), D_{x} \varphi\left(\xi_{\varepsilon}, \zeta_{\varepsilon}\right), X\right) \in \overline{\mathscr{P}}^{2,+} u\left(x_{\varepsilon}, t_{\varepsilon}\right) \\
\left(-\varphi_{s}\left(\xi_{\varepsilon}, \zeta_{\varepsilon}\right),-D_{y} \varphi\left(\xi_{\varepsilon}, \zeta_{\varepsilon}\right), Y\right) \in \overline{\mathscr{P}}^{2,-} v\left(y_{\varepsilon}, s_{\varepsilon}\right)
\end{gathered}
$$

the closed parabolic super- and subjets, and

$$
-\left(\frac{1}{\lambda}+\left|A_{0}\right|\right) I \leq\left(\begin{array}{cc}
X & O \\
O & -Y
\end{array}\right) \leq A_{0}+\lambda A_{0}^{2}
$$

where

$$
A_{0}=\left(\begin{array}{ll}
D_{x x}^{2} \varphi\left(x_{\varepsilon}, t_{\varepsilon}, y_{\varepsilon}, s_{\varepsilon}\right) & D_{x y}^{2} \varphi\left(x_{\varepsilon}, t_{\varepsilon}, y_{\varepsilon}, s_{\varepsilon}\right) \\
D_{y x}^{2} \varphi\left(x_{\varepsilon}, t_{\varepsilon}, y_{\varepsilon}, s_{\varepsilon}\right) & D_{y y}^{2} \varphi\left(x_{\varepsilon}, t_{\varepsilon}, y_{\varepsilon}, s_{\varepsilon}\right)
\end{array}\right) .
$$

Using again the fact that $u$ and $v$ are, respectively, suband supersolution of (20) and that $D_{x} \varphi\left(x_{\varepsilon}, t_{\varepsilon}, y_{\varepsilon}, s_{\varepsilon}\right)=$ $-D_{y} \varphi\left(x_{\varepsilon}, t_{\varepsilon}, y_{\varepsilon}, s_{\varepsilon}\right) \neq 0$, we have by the equation of $u$

$$
\begin{gathered}
\alpha+\gamma\left(t_{\varepsilon}-s_{\varepsilon}\right)+f_{*}\left(x_{\varepsilon}\right)\left|D_{x} \varphi\left(x_{\varepsilon}, t_{\varepsilon}, y_{\varepsilon}, s_{\varepsilon}\right)\right| \\
+F\left(D_{x} \varphi\left(x_{\varepsilon}, t_{\varepsilon}, y_{\varepsilon}, s_{\varepsilon}\right), X\right) \leq 0
\end{gathered}
$$

and by the equation of $v$

$$
\begin{gathered}
\gamma\left(t_{\varepsilon}-s_{\varepsilon}\right)+f^{*}\left(y_{\varepsilon}\right)\left|-D_{y} \varphi\left(x_{\varepsilon}, t_{\varepsilon}, y_{\varepsilon}, s_{\varepsilon}\right)\right| \\
+F\left(-D_{y} \varphi\left(x_{\varepsilon}, t_{\varepsilon}, y_{\varepsilon}, s_{\varepsilon}\right), Y\right) \geq 0 .
\end{gathered}
$$

Adding these two inequalities we obtain

$$
\begin{aligned}
\alpha \leq & \left(f^{*}\left(y_{\varepsilon}\right)-f_{*}\left(x_{\varepsilon}\right)\right)\left|D_{x} \varphi\left(x_{\varepsilon}, t_{\varepsilon}, y_{\varepsilon}, s_{\varepsilon}\right)\right| \\
& +F\left(-D_{y} \varphi, Y\right)-F\left(D_{x} \varphi, X\right) .
\end{aligned}
$$

Since

$$
\begin{aligned}
D_{x x}^{2} \varphi\left(x_{\varepsilon}, t_{\varepsilon}, y_{\varepsilon}, s_{\varepsilon}\right) & =D_{y y}^{2} \varphi\left(x_{\varepsilon}, t_{\varepsilon}, y_{\varepsilon}, s_{\varepsilon}\right) \\
& =-D_{x y}^{2} \varphi\left(x_{\varepsilon}, t_{\varepsilon}, y_{\varepsilon}, s_{\varepsilon}\right) \\
& =-D_{y x}^{2} \varphi\left(x_{\varepsilon}, t_{\varepsilon}, y_{\varepsilon}, s_{\varepsilon}\right)
\end{aligned}
$$

multiplying the second matrix inequality in (41) twice by vectors of the form $(\xi, \xi) \in \mathbb{R}^{n+1} \times \mathbb{R}^{n+1}$ implies $X \leq Y$. Therefore since $F$ is elliptic,

$$
\begin{aligned}
F & \left(-D_{y} \varphi\left(x_{\varepsilon}, t_{\varepsilon}, y_{\varepsilon}, s_{\varepsilon}\right), Y\right) \\
& =F\left(D_{x} \varphi\left(x_{\varepsilon}, t_{\varepsilon}, y_{\varepsilon}, s_{\varepsilon}\right), Y\right) \\
& \leq F\left(D_{x} \varphi\left(x_{\varepsilon}, t_{\varepsilon}, y_{\varepsilon}, s_{\varepsilon}\right), X\right)
\end{aligned}
$$

and the inequality in (45) simply becomes

$$
\begin{aligned}
\alpha & \leq\left(f^{*}\left(y_{\varepsilon}\right)-f_{*}\left(x_{\varepsilon}\right)\right)\left|D_{x} \varphi\left(x_{\varepsilon}, t_{\varepsilon}\right)\right| \\
& =\frac{\gamma}{\varepsilon}\left(f^{*}\left(y_{\varepsilon}\right)-f_{*}\left(x_{\varepsilon}\right)\right)\left|\frac{x_{\varepsilon}-y_{\varepsilon}}{\varepsilon}+\eta\right|^{3} .
\end{aligned}
$$

Now we analyze the right hand side of (48) as $\varepsilon \rightarrow 0$. If $\bar{x} \in \Gamma$ and $x_{\varepsilon} \in \Omega^{-} \cup \Gamma$ then (31) implies that also $y_{\varepsilon} \in \Omega^{-}$, for all $\varepsilon$ sufficiently small as we already observed. Inequality (48) and Lipschitz continuity of $f$ in the region $\Omega^{-}$therefore yield

$$
\alpha \leq \gamma L_{f} \frac{\left|x_{\varepsilon}-y_{\varepsilon}\right|}{\varepsilon}\left|\frac{x_{\varepsilon}-y_{\varepsilon}}{\varepsilon}+\eta\right|^{3},
$$

where $L_{f}$ is a Lipschitz constant for $f$ in $\Omega^{-}$. Notice that the same estimate holds true if $\bar{x} \notin \Gamma$ as $f$ is Lipschitz continuous in a neighborhood of $\bar{x}$. Finally, letting $\varepsilon \rightarrow 0^{+}$and using (30) we get a contradiction in these cases since the right hand side of (49) tends to 0 as $\varepsilon \rightarrow 0^{+}$.

We are left with the case $\bar{x} \in \Gamma$ and $x_{\varepsilon} \in \Omega^{+}$along a subsequence. We have two further subcases: either for $\varepsilon$ small $y_{\varepsilon} \in \Omega^{+} \cup \Gamma$ and we proceed similarly as above by the Lipschitz continuity of $f$ in the region $\Omega^{+}$or for $y_{\varepsilon} \in \Omega^{-}$on a subsequence. In the latter situation we observe that

$$
\lim _{\varepsilon \rightarrow 0^{+}}\left[f\left(y_{\varepsilon}\right)-f\left(x_{\varepsilon}\right)\right]=\left[f_{*}(\bar{x})-f^{*}(\bar{x})\right]<0
$$

and again we obtain a contradiction in (48) for $\varepsilon$ small ending the proof.

An immediate consequence of the previous result is the uniqueness of solutions of (1).

Corollary 5. Assume that $f: \Omega \rightarrow \mathbb{R}$ is a piecewise continuous function across a Lipschitz graph. Let $u: \bar{\Omega}_{\infty} \rightarrow \mathbb{R}$ be a continuous viscosity solution of (1). Then $u$ is the unique solution of (1) within the class of possibly discontinuous viscosity solutions $v: \bar{\Omega}_{\infty} \rightarrow \mathbb{R}$ which are continuous at the points of the parabolic boundary. 
Proof. Just apply the theorem to $u$ and $v_{*}$ first and then to $u$ and $v^{*}$. Therefore $v^{*} \leq u \leq v_{*}$ in $\Omega_{\infty}$; hence $v$ is continuous as well and $u=v$.

\section{Competing Interests}

The authors declare that they have no competing interests.

\section{References}

[1] M. G. Crandall, H. Ishii, and P.-L. Lions, "User's guide to viscosity solutions of second order partial differential equations," Bulletin of the American Mathematical Society, vol. 27, no. 1, pp. 1-67, 1992.

[2] S. Osher and J. A. Sethian, "Fronts propagating with curvaturedependent speed: algorithms based on Hamilton-Jacobi formulations," Journal of Computational Physics, vol. 79, no. 1, pp. 1249, 1988.

[3] H. Ishii, "Hamilton-Jacobi equations with discontinuous Hamiltonians on arbitrary open sets," Bulletin of the Faculty of Science and Engineering, Chuo University, vol. 28, pp. 33-77, 1985.

[4] R. R. Jensen, "Uniformly elliptic PDEs with bounded, measurable coefficients," The Journal of Fourier Analysis and Applications, vol. 2, no. 3, pp. 237-259, 1996.

[5] L. A. Caffarelli, M. G. Crandall, M. J. Kocan, and A. J. Święch, "On viscosity solutions of fully nonlinear equations with measurable ingredients," Communications on Pure and Applied Mathematics, vol. 49, no. 4, pp. 365-397, 1996.

[6] F. Camilli and A. Siconolfi, "Hamilton-Jacobi equations with measurable dependence on the state variable," Advances in Differential Equations, vol. 8, no. 6, pp. 733-768, 2003.

[7] P. Soravia, "Boundary value problems for Hamilton-Jacobi equations with discontinuous Lagrangian," Indiana University Mathematics Journal, vol. 51, no. 2, pp. 451-477, 2002.

[8] P. Soravia, "Degenerate eikonal equations with discontinuous refraction index," ESAIM. Control, Optimisation and Calculus of Variations, vol. 12, no. 2, pp. 216-230, 2006.

[9] P. Soravia, "Uniqueness results for fully nonlinear degenerate elliptic equations with discontinuous coefficients," Communications on Pure and Applied Analysis, vol. 5, no. 1, pp. 213-240, 2006.

[10] C. De Zan and P. Soravia, "Cauchy problems for noncoercive Hamilton-Jacobi-Isaacs equations with discontinuous coefficients," Interfaces and Free Boundaries, vol. 12, no. 3, pp. 347$368,2010$.

[11] X. Chen and B. Hu, "Viscosity solutions of discontinuous Hamilton-Jacobi equations," Interfaces and Free Boundaries, vol. 10, no. 3, pp. 339-359, 2008.

[12] K. Deckelnick and C. M. Elliott, "Uniqueness and error analysis for Hamilton-Jacobi equations with discontinuities," Interfaces and Free Boundaries, vol. 6, no. 3, pp. 329-349, 2004.

[13] G. Barles, A. Briani, and E. Chasseigne, "A Bellman approach for two-domains optimal control problems in $\mathbb{R}^{N}$, ESAIM: Control, Optimisation and Calculus of Variations, vol. 19, no. 3 , pp. 710-739, 2013.

[14] G. Barles, A. Briani, and E. Chasseigne, "A Bellman approach for regional optimal control problems in $\mathbb{R}^{n}$," SIAM Journal on Control and Optimization, vol. 52, no. 3, pp. 1712-1744, 2014.

[15] A. Bressan and Y. Hong, "Optimal control problems on stratified domains," Networks and Heterogeneous Media, vol. 2, no. 2, pp. 313-331, 2007.
[16] G. M. Coclite and N. H. Risebro, "Viscosity solutions of Hamilton-Jacobi equations with discontinuous coefficients," Journal of Hyperbolic Differential Equations, vol. 4, no. 4, pp. 771-795, 2007.

[17] L. C. Evans and J. Spruck, "Motion of level sets by mean curvature I," Journal of Differential Geometry, vol. 33, pp. 635-681, 1991.

[18] Y.-G. Chen, Y. Giga, and S. Goto, "Uniqueness and existence of viscosity solutions of generalized mean curvature flow equations," Journal of Differential Geometry, vol. 33, no. 3, pp. 749786, 1991.

[19] H. Ishii and P. E. Souganidis, "Generalized motion of noncompact hypersurfaces with velocity having arbitrary growth on the curvature tensor," The Tohoku Mathematical Journal, vol. 47, no. 2, pp. 227-250, 1995.

[20] S. Goto, "Generalized motion of hypersurfaces whose growth speed depends superlinearly on the curvature tensor," Differential and Integral Equations, vol. 7, no. 2, pp. 323-343, 1994.

[21] Y. Giga, Surface Evolution Equations. A Level Set Approach, vol. 99 of Monographs in Mathematics, Birkhäuser, Basel, Switzerland, 2006.

[22] P. E. Souganidis, "Front propagation: theory and applications," in Viscosity Solutions and Applications, M. Bardi, M. G. Crandall, L. C. Evans, H. M. Soner, and P. E. Souganidis, Eds., vol. 1660 of Lecture Notes in Mathematics, pp. 186-242, Springer, Berlin, Germany, 1997.

[23] M. Bardi and P. Soravia, "A comparison result for HamiltonJacobi equations and applications to some differential games lacking controllability," Funkcialaj Ekvacioj, vol. 37, pp. 19-43, 1994. 


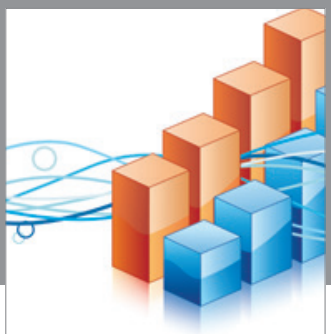

Advances in

Operations Research

vatem alat4

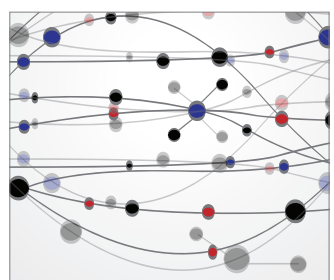

\section{The Scientific} World Journal
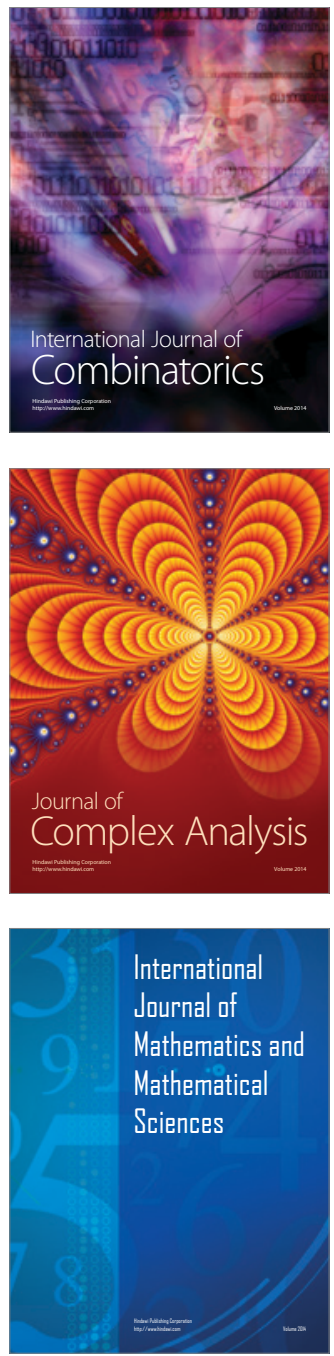
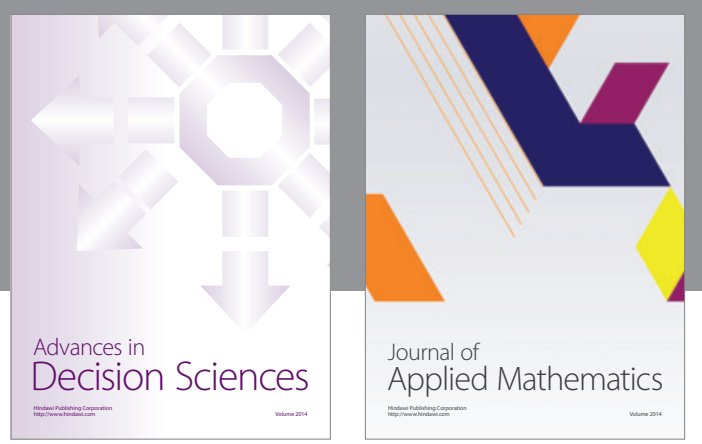

Algebra

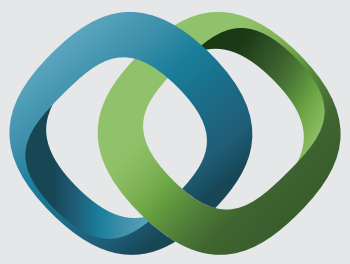

\section{Hindawi}

Submit your manuscripts at

http://www.hindawi.com
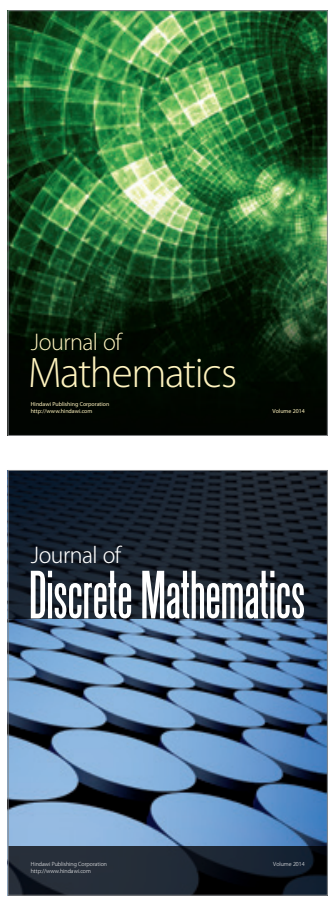

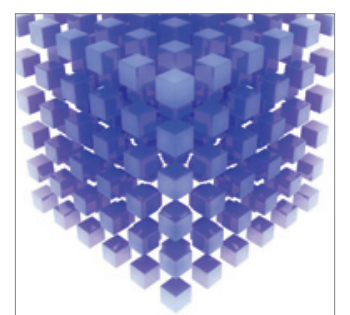

Mathematical Problems in Engineering
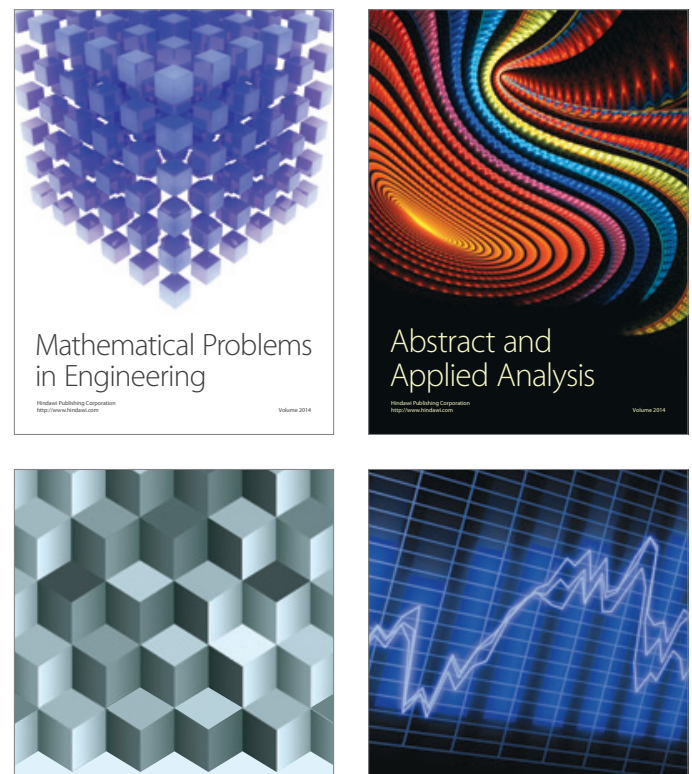

Journal of

Function Spaces

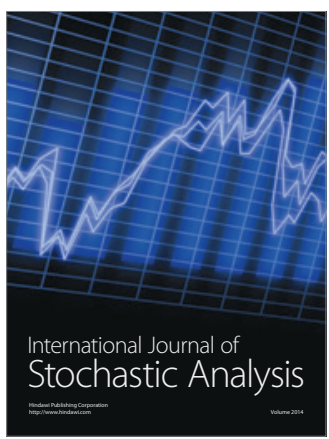

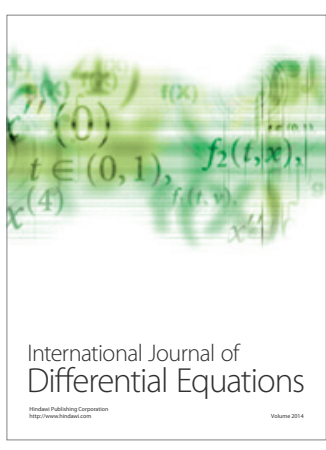
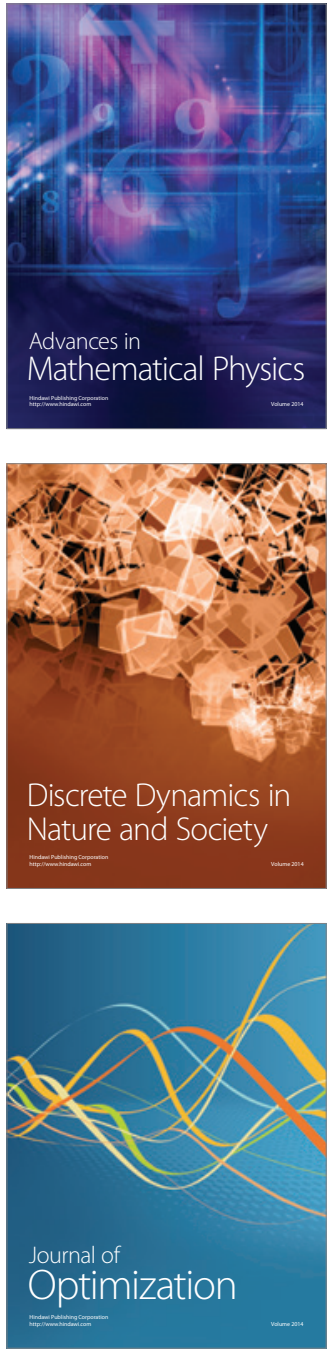